\title{
The study of a fourth-order differential equation: existence, uniqueness and a dynamical system approach
}

\author{
Cristian - Paul Danet ${ }^{1, *}$ \\ ${ }^{1}$ Department of Applied Mathematics \\ University of Craiova \\ Al. I. Cuza St., 13, 200585 Craiova, Romania
}

\begin{abstract}
This paper is concerned with the problem of existence and uniqueness of solutions for the semilinear fourth-order differential equation $u^{i v}-k u^{\prime \prime}+$ $a(x) u+c(x) f(u)=0$. Existence and uniqueness is proved using variational methods and maximum principles. We also give a dynamical system approach to the equation. We study the bifurcation of the system and show that the behaviour of the stationary points $S(\alpha, 0,0,0)$ depend on the relation between the parameter $k$ and $\beta=f^{\prime}(\alpha)$.
\end{abstract}

\section{Introduction}

This paper deals with the boundary value problem (1)

$$
\left\{\begin{array}{l}
u^{i v}-k u^{\prime \prime}+a(x) u+c(x) f(u)=0 \text { in } \Omega, \\
u=u^{\prime \prime}=0 \text { on } \partial \Omega
\end{array}\right.
$$

Here $c \in C^{0}(\bar{\Omega}), c_{M} \geq c(x) \geq c_{m}, c_{M}>0, f \in C^{0}(\Omega), a(x) \geq 0$ is bounded in $\Omega=\left[L_{1}, L_{2}\right]$ and $k$ is a constant.

The equation (1) (with various nonlinearities $f(u)$ ) arises in several contexts:

- the waves on a suspension bridge [6], [18] $\left(f(u)=(u+1)^{+}-1\right)$

- travelling water waves in a shallow channel [4] $\left(f(u)=u-u^{2}\right)$

- the buckling of a strut on a nonlinear elastic foundation [1], [20] $\left(f(u)=u-u^{3}+\alpha u^{5}, \alpha \geq\right.$ 1/4)

- when $\varphi \equiv k$ ( $k$ const. $<0)$, and $f(u)=u^{3}-u,(1)$ is known as Fisher - Kolmogorov equation, and appears in population dynamics.

We also note that the extension of (1), to the higher dimensional case $(n \geq 2)$ reads

$$
\Delta^{2} u+\varphi(x) \Delta u+f(u)=0 \text { in } \Omega \subset \mathbb{R}^{\mathrm{n}}, \mathrm{n} \geq 2,
$$

and also serves as a model of some applications: [14]

- the case $\varphi \equiv 0$ and $f(u)=k_{1} u^{3}+k_{2} u, k_{1}, k_{2}>0$ arises in bending of cylindrical shells

- the case $\varphi \equiv 0$ and $f(u)=-k u, k>0$, arises in thin plate theory [16]

\footnotetext{
*e-mail: cristiandanet@yahoo.com
} 
- the case $\varphi \equiv 0, f(u)=e^{u}$ represents a natural higher order extension of the celebrated equation $-\Delta u=e^{u}$, which describes the problem of self - ignition [12].

The purpose of this work is to study via variational methods and using maximum principles the existence and uniqueness of (1). We obtain, in some sense, generalizations of results obtained in Peletier et al. ([19]), where (1) is considered with $a(x)=-k$ and $f(u)=k u^{3}$ or generalizations of results presented in the paper of Tersian and Chaparova ([22]), where the nonlinearity $f(u)=b(x) u^{3}$.

We are able to treat the a general case when

$$
F(s) \geq-K_{1}|s|^{r}-K_{2}, \text { where } K_{1}>0, K_{2}>0, r \geq 1,
$$

where $F(u)=\int_{0}^{u} f(s) d s$.

We also give a dynamical system approach of equation (1). We show that if there exists $\alpha$ such that $f(\alpha)=0$ then the point $S(\alpha, 0,0,0)$ is an equilibrium point for the associated dynamical system. The behaviour of $S(\alpha, 0,0,0)$ depends on the relation between the parameter $k$ and $\beta=f^{\prime}(\alpha)$.

Throughout the paper $C$ denotes a universal positive constant unless otherwise speci-fied.

\section{Variational Settings and Preliminaries}

Definition 2.1 Let $H(\Omega)=W^{2,2}(\Omega) \cap W_{0}^{1,2}(\Omega)$.

A weak solution of $(1)$ is a function $u \in H(\Omega)$ such that

$$
\int_{\Omega}\left(u^{\prime \prime} v^{\prime \prime}+k u^{\prime} v^{\prime}+a(x) u v+c(x) f v\right) d x=0 \quad \forall v \in H(\Omega) .
$$

A classical solution of (1) is a function $u \in \mathrm{C}^{4}(\Omega)$ that satisfies (1).

Note that a weak solution of (1) is also a classical solution of (1) (for a proof see [22]).

The problem (1) has a variational structure and the weak solutions in the space $H(\Omega)$ can be found as critical points of the functional

$$
\begin{gathered}
J: H(\Omega) \rightarrow \mathbb{R} \\
J(u)=\frac{1}{2} \int_{\Omega}\left(\left(u^{\prime \prime}\right)^{2}+k\left(u^{\prime}\right)^{2}+a(x) u^{2}\right) d x+\int_{\Omega} c(x) F(u) d x,
\end{gathered}
$$

which is Fréchet - differentiable and its Fréchet derivative is given by

$$
<J^{\prime}(u), v>=\int_{\mathbb{R}}\left(u^{\prime \prime} v^{\prime \prime}+k u^{\prime} v^{\prime}+a u v+c(x) f(u) v\right) d x \forall v \in H(\Omega) .
$$

The following lemma will be useful.

Lemma 2.1 There exist a constant $C_{1}>0$ such that for all $u \in H(\Omega)$

$$
\int_{\Omega}\left[\left(u^{\prime \prime}\right)^{2}-k\left(u^{\prime}\right)^{2}+a(x) u^{2}\right] d x \geq C_{1}\|u\|_{W^{2,2}(\Omega)}^{2},
$$


where

$$
\|u\|_{W^{2,2}(\Omega)}^{2}=\int_{\Omega}\left[u^{2}+\left(u^{\prime}\right)^{2}+\left(u^{\prime \prime}\right)^{2}\right] d x,
$$

and $0 \leq k \leq 2 \sqrt{a_{m}}, a(x) \geq a_{m}>0$ in $\Omega$. Here $C_{1}=\min \left(-k, a_{m}, 1\right)$ if $k<0$. If $k>0$ then $C_{1}=1-C_{2} / 3$, where

$$
C_{2}=1-k-2 a_{m}+\sqrt{\left(k+2 a_{m}-1\right)^{2}+3(k+1)^{2}} .
$$

The proof follows from Lemma 8, [22].

\section{Existence and Uniqueness Results}

Case $0<r<2$

It is well known (see [17], Chapter 1) that $H(\Omega)$ becomes a Hilbert space endowed with the scalar product

$$
(u, v) \rightarrow \int_{\Omega} u^{\prime \prime} v^{\prime \prime} d x, \quad u, v \in H(\Omega) .
$$

This scalar product induces a norm denoted $\|\cdot\|_{1}$ equivalent to $\|\cdot\|_{W^{2,2}(\Omega)}$.

Lemma 3.1 Suppose that $F$ satisfies (3), $k \geq 0$. Then there exists a minimizer $\tilde{u} \in H(\Omega)$ of $J$. If in addition we admit that $f^{\prime} \geq 0$ in $\mathbb{R}$, then the minimizer is unique.

Proof. Let us first establish that $J$ is coercive.

By the Cauchy inequality

$$
F(u) \geq-\epsilon u^{2}-C(\epsilon, r) K_{1}^{\frac{2}{2-r}}-K_{2}, \quad \text { where } \epsilon>0 .
$$

By Lemma 1, inequality (b), [23] and since the norms $\|\cdot\|_{1},\|\cdot\|_{W^{2,2}(\Omega)}$ are equivalent we obtain that for all $u \in W^{2,2}(\Omega) \cap W_{0}^{1,2}(\Omega)$

$$
\int_{\Omega} u^{2} d x \leq \frac{L^{4}}{\pi^{4}} \int_{\Omega}\left(u^{\prime \prime}\right)^{2} d x=\frac{L^{4}}{\pi^{4}}\|u\|_{1}^{2} \leq C(L)\|u\|_{W^{2,2}(\Omega)}^{2},
$$

where $L=L_{2}-L_{1}$.

Consequently by (4) and (5) we get

$$
J(u) \geq C(L, k)\|u\|_{W^{2,2}(\Omega)}^{2}-\varepsilon C\left(L, c_{M}\right)\|u\|_{W^{2,2}(\Omega)}^{2}-C\left(\varepsilon, r, c_{M}, L, K_{2}\right) .
$$

Hence $J$ is coercive if we choose $\varepsilon$ sufficiently small.

We now show that $J(u)$ is weakly lower semicontinous on the reflexive space $W^{2,2}(\Omega)$.

Since $k \geq 0$ we get that

$$
J_{1}(u)=\frac{1}{2} \int_{\Omega}\left(\left(u^{\prime \prime}\right)^{2}+k\left(u^{\prime}\right)^{2}\right) d x
$$

is convex.

Hence $J(u)$ can be represented as the sum $J(u)=J_{1}(u)+J_{2}(u)$, where $J_{1}(u)$ is convex and $J_{2}(u)=\int_{\Omega}\left(a(x) u^{2}+c(x) F(u)\right) d x$ is (sequentially) weakly continuous. 
Therefore, $J(u)$ is weakly lower semicontinous by Criterion 6.1.3 in [2], p.301.

We obtain thus the existence of the minimizer as required.

If $f^{\prime} \geq 0$ then $J(u)$ is convex and the uniqueness of the minimizer follows.

\section{Case $r=2$}

According to Lemma 2.1 the space $H(\Omega)$ endowed with the scalar product

$$
(u, v)=\int_{\Omega}\left[u^{\prime \prime} v^{\prime \prime}+k u^{\prime} v^{\prime}+a(x) u v\right] d x
$$

becomes a Hilbert space $(k \in \mathbb{R})$.

This scalar product generates a norm denoted $\|\cdot\|_{2}$

$$
\|u\|_{2}^{2}=(u, u)=\int_{\Omega}\left(\left(u^{\prime \prime}\right)^{2}+k\left(u^{\prime}\right)^{2}+a(x) u^{2}\right) d x .
$$

Hence $J$ becomes

$$
J(u)=\frac{1}{2}\|u\|_{2}^{2}+\int_{\Omega} c(x) F(u) d x .
$$

Lemma 3.2 Suppose that F satisfies (3). If in addition we admit that

$$
1-\frac{2 K_{1} C_{M}}{C_{1}}>0
$$

then the boundary value problem (1) has at least a weak solution.

The proof uses the following inequality (which is a consequence of Lemma 2.1)

$$
\int_{\Omega} u^{2} d x \leq \frac{1}{C_{1}}\|u\|_{2}^{2}
$$

and is similar to the proof of Lemma 2.1

The following result ensures that the solution we have found is nontrivial.

Lemma 3.3 Suppose that one of the following conditions hold:

$$
\lim _{s \rightarrow \pm \infty} \frac{f(s)}{s^{\alpha}}=\beta, \beta \neq 0, \text { and } \lim _{s \rightarrow \infty} F(s)=\infty \quad\left(\text { or } \lim _{\mathrm{s} \rightarrow-\infty} \mathrm{F}(\mathrm{s})=-\infty\right),
$$

where $\alpha \geq 2, c \neq 0$ and

- $\beta<0$ if $c(x) \geq 0$ in $\Omega$

or

- $\beta>0$ if $c(x) \leq 0$ in $\Omega$.

Then there exists $e \in H(\Omega)$ such that $J(e)<0$. 
Proof.

According to the existence theory of ODE, there exists a function $\varphi=0$ on $\partial \Omega, \varphi \in$ $C^{2}(\bar{\Omega}) \subset H(\Omega)$ such that $\varphi>0$ in $\Omega$.

$$
\begin{aligned}
\lim _{s \rightarrow \infty} \frac{J(s \varphi)}{s^{\alpha+1}} & =\frac{1}{2} \lim _{s \rightarrow \infty} \frac{s^{2}\|\varphi\|_{2}^{2}}{s^{\alpha+1}}+\lim _{s \rightarrow \infty} \int_{\Omega} \frac{c(x) F(s \varphi)}{s^{\alpha+1}} d x \\
& =\int_{\Omega} \lim _{s \rightarrow \infty} \frac{c(x) F(s \varphi)}{s^{\alpha+1}} d x=\int_{\Omega} \lim _{s \rightarrow \infty} \frac{c(x) f(s \varphi) \varphi}{(\alpha+1) s^{\alpha}} d x \\
& =\frac{\beta}{\alpha+1} \int_{\Omega} c(x) \varphi^{\alpha} d x<0 .
\end{aligned}
$$

Hence there exists $e=s \varphi \in H(\Omega)$ such that $J(e)<0$.

We are now able to prove our main result.

THEOREM 3.1 Suppose that we are either under the hypotheses of Lemma 3.1 and Lemma 3.3 or Lemma 3.2 and Lemma 3.3.

Then the boundary value problem (1) has at least one nontrivial solution. If $f^{\prime} \geq 0$ in $\mathbb{R}$ then, the solution is unique.

If in addition $F \geq 0$ in $\mathbb{R}$ then the unique solution is the trivial one.

Proof. The existence part of the theorem follows from Lemma 3.1, since the minimizer is the solution to (1).

From Lemma 3.3 follows that the solution in nontrivial.

$f^{\prime} \geq 0$ and $F \geq 0$ implies $f(0)=0$ and hence $u \equiv 0$ is the unique solution.

\section{Case $r>2$.}

The proof (for a proof see [10]) is long and is based on the following four lemmas and on a version of the celebrated Mountain-Pass Theorem due to Brezis and Nirenberg ([3],[11]). We just state the results without proof.

Theorem 3.2 (Mountain-Pass Theorem [3], [11]) Let E be a real Banach space with its dual $E^{*}$ and suppose that $J \in C^{1}(E, \mathbb{R})$ satisfies

$$
\max \{J(0), J(e)\} \leq \mu<\eta \leq \inf _{\|u\|=\rho} J(u),
$$

for some constants $\mu<\eta, \rho>0$ and $e \in E$ with $\|e\|>\rho$. Let $\delta \geq \eta$ be characterized by

$$
\delta=\inf _{\gamma \in \Gamma} \max _{0 \leq \tau \leq 1} J(\gamma(\tau))
$$

where $\Gamma=\left\{\gamma \in C^{0}([0,1], E): \gamma(0)=0, \gamma(1)=e\right\}$ is the set of continuous path joining 0 and $e$.

Then there exists a sequence $\left\{u_{n}\right\} \subset E$ such that

$$
J\left(u_{n}\right) \rightarrow \delta \geq \eta \quad \text { and } \quad\left\|J^{\prime}\left(u_{n}\right)\right\|_{E^{*}} \rightarrow 0, \quad \text { as } n \rightarrow \infty .
$$

The following two lemmas show that the functional $J$ has the mountain pass geometry.

Lemma 3.4 Let F satisfy(3). Then there exist two positive constants $\rho$ and $\eta$ such that

$$
J(u)_{\|_{\|u\|_{2}=\rho}} \geq \eta .
$$




\section{Lemma 3.5 Suppose that}

$$
\lim _{s \rightarrow \infty} \frac{f(s)}{s^{\alpha}}=\beta, \beta \neq 0 \text { and } \lim _{s \rightarrow \infty} F(s)=\infty,
$$

where $\bullet>3$, odd if $\beta>0$;

- $\alpha \geq 2$ if $\beta<0$.

Then there exists $e \in H(\Omega)$ with $\|e\|_{2}>\rho$ such that $J(e)<0$.

Remark. Lemma 3.5 still holds if we replace $\infty$ by $-\infty$ in (7). i.e.,

We can now apply the Mountain-Pass Theorem in $H(\Omega)$ to find a Cerami type sequence,

$$
\text { there exists }\left\{u_{n}\right\} \subset H(\Omega) \text { such that } J\left(u_{n}\right) \rightarrow \delta \text { and }\left\|J^{\prime}\left(u_{n}\right)\right\|_{H^{*}(\Omega)} \rightarrow 0 \text {. }
$$

Lemma 3.6 Suppose that we are under the hypotheses of Lemma 3.4 and Lemma 3.5. If there exist the constants $K>0, \theta \geq 3$, and $0<\gamma<2$ such that

$$
F(s)-\frac{1}{\theta} f(s) s \geq-K s^{\gamma}, s>0,
$$

then the sequence $\left\{u_{n}\right\}$ defined by $(8)$ is bounded in $H(\Omega)$.

Remark. Lemma 3.6 still holds when $\gamma=2$, if we impose some restrictions.

Lemma 3.7 Under the hypotheses of Lemma 3.6, there exists a sequence $\left\{u_{n}\right\}$ such that $u_{n} \rightarrow$ $u_{0}$ strongly in $H(\Omega)$.

An immediate consequence of Lemma 3.7 is the existence result:

THEOREM 3.3 The problem (1) admits a nontrivial solution in $H(\Omega)$.

The following theorem collects several results and shows that uniqueness results may hold even if the coefficient of $u$ is unbounded, nonsmooth or has arbitrary sign.

THEOREM 3.4 If one of the following conditions is satisfied

1.([9])

$$
k=k(x)>0 \text { in } \bar{\Omega},\left(a f^{\prime}\right)^{\prime \prime}<0 \text { in } \Omega,
$$

2. ([9])

$$
k>0(\text { constant }), a f^{\prime}>0 \text { in } \bar{\Omega}, \mathrm{k}^{2} \geq\left(\frac{\mathrm{af}^{\prime}-1}{\mathrm{af}^{\prime}}\right)^{2} \text { in } \Omega,
$$

In particular, the result holds if $k>1$. 
3.

$$
k>0(\text { constant }), a f^{\prime}>0 \text { in } \bar{\Omega}, \sup _{\Omega}\left(\mathrm{k}-\frac{1}{\mathrm{k}}\left(\frac{\mathrm{af}^{\prime}-1}{\mathrm{af}^{\prime}}\right)^{2}\right)<\frac{4}{\mathrm{~L}^{2} \mathrm{e}^{2}},
$$

4.

$$
\max \left\{2, \sup _{\Omega}\left(a f^{\prime}\right)^{2}+1\right\}<\frac{4}{L^{2} e^{2}},
$$

$k=k(x) \geq 0$ in $\Omega$ and a $f^{\prime}$ is of arbitrary sign in $\Omega$,

then there exists at most one classical solution of

$$
\left\{\begin{array}{l}
u^{i v}-k(x) u^{\prime \prime}+a(x) f(u)=0 \text { in } \Omega \\
u=g_{1}, u^{\prime \prime}=g_{2} \text { on } \partial \Omega
\end{array}\right.
$$

Proof.

We prove the uniqueness result under the assumption (12).

It suffices to show that $u \equiv 0$ the unique solution of the homogenous, linear boundary value problem $\left(\rho=a(x) f^{\prime}\right)$.

$$
\left\{\begin{array}{l}
u^{i v}-k(x) u^{\prime \prime}+\rho(x) u=0 \text { in } \Omega \\
u=u^{\prime \prime}=0 \text { on } \partial \Omega
\end{array}\right.
$$

Let

$$
\mathrm{P}=\frac{1}{2}\left(u^{\prime \prime}-\rho u\right)^{2}+\frac{1}{2}\left(u^{\prime \prime}\right)^{2}+u^{2},
$$

where $u$ is a solution of (15). By computing, we see that for a constant $\gamma>0$ we get

$$
\mathrm{P}^{\prime \prime}+\gamma \mathrm{P} \geq 0 \quad \text { in } \Omega \text {. }
$$

By the generalized maximum principle, Theorem 10, p.73, [21], there exists a real constant such that

$$
\frac{\mathrm{P}}{w} \equiv \text { constant in } \bar{\Omega}
$$

or

$$
\frac{\mathrm{P}}{w} \text { does not attain a nonnegative maximum in } \Omega \text {. }
$$

Here $w>0$ in $\bar{\Omega}$ is a smooth function (see for details [21], [8], [9]).

The function $\mathrm{P} / w$ is smooth and hence (16) holds in $\bar{\Omega}$.

By the boundary conditions we have $\mathrm{P}=0$ on $\partial \Omega$, i.e., the constant $=0$. It follows that $\mathrm{P}=0$ in $\Omega$, which means $u \equiv 0$ in $\Omega$.

We are left to check the condition (17), i.e.,

$$
\max _{\bar{\Omega}} \frac{\mathrm{P}}{w}=\max _{\partial \Omega} \frac{\mathrm{P}}{w} .
$$

By the boundary conditions we have

$$
0 \leq \max _{\bar{\Omega}} \frac{\mathrm{P}}{w}=0
$$


i.e., $u \equiv 0$ in $\Omega$.

Note that the uniqueness results 10 and 11 hold in $n$ dimensions and extend (in some sense) a classical result of Chow and Dunninger [5].

For further uniqueness results (in higher dimensions) the reader is referred to the paper [7] and [8].

\section{A Dynamical System Approach}

To give a dynamical approach to (1), we consider the following version of (1) in $\mathbb{R}$

$$
u^{i v}+k u^{\prime \prime}+f(u)=0
$$

and rewrite (18) as a dynamical system (a fourth order system of four equations)

$$
\left\{\begin{array}{l}
x_{1}^{\prime}=x_{2}, \\
x_{2}^{\prime}=x_{3}, \\
x_{3}^{\prime}=x_{4}, \\
x_{4}^{\prime}=-k x_{3}-f\left(x_{1}\right) .
\end{array}\right.
$$

Let us define $F: \mathbb{R}^{4} \rightarrow \mathbb{R}^{4}$ by $F\left(x_{1}, x_{2}, x_{3}, x_{4}\right)=\left(x_{2}, x_{3}, x_{4},-k x_{3}-f\left(x_{1}\right)\right.$.

Then the system (19) may be rewritten as

$$
X^{\prime}(t)=F(X(t))
$$

where a solution to (19) has the form $X(t)=\left(x_{1}(t), x_{2}(t), x_{3}(t), x_{4}(t)\right)$.

Suppose that $f$ has a zero at $\alpha$. Then the dynamical system (19) admits a stationary point which is $S=(\alpha, 0,0,0)$. Note that this point corresponds to the solution $u \equiv \alpha$ to (18).

We intend to study the dynamical system (19)

Assume that $f \in C^{1}(\mathbb{R})$ and $f^{\prime}(\alpha)=\beta$. Then the linearized problem for (19) reads

$$
X^{\prime}(t)=A X(t)
$$

where

$$
A=\left[\begin{array}{cccc}
0 & 1 & 0 & 0 \\
0 & 0 & 1 & 0 \\
0 & 0 & 0 & 1 \\
-\beta & 0 & -k & 0
\end{array}\right]
$$

The eigenvalues of $A$ satisfy the equation $\lambda^{4}+k \lambda^{2}+\beta=0$.

We can prove:

THEOREM 4.1 The following cases occur:

Case $\beta>0$.

- if $k<-2 \sqrt{\beta}$ then the eigenvalues of $A$ are all real and distinct, i.e. the dynamical system (19) has a stationary point $S(\alpha, 0,0,0)$ which is a real saddle, and A has a 2 - dimensional stable manifold and a 2 - dimensional unstable manifold, both not oscillating near $S$.

- if $-2 \sqrt{\beta}<k<2 \sqrt{\beta}$ then all eigenvalues of $A$ are complex, i.e., $S$ is a saddle focus and $S$ has a 2 - dimensional stable manifold and a 2 - dimensional unstable manifold. 

center.

- if $k>2 \sqrt{\beta}$ then the eigenvalues of $A$ are purely complex and distinct and hence $S$ is a

- if $k=2 \sqrt{\beta}$ then eigenvalues of $A$ are purely complex but not distinct (double).

- if $k=-2 \sqrt{\beta}$ then eigenvalues of $A$ are real but not distinct (double).

Case $\beta<0$.

- if $\beta<0$ ( $k$ real) then $A$ has 2 real eigenvalues $\lambda_{1}=\lambda_{1}(k, \beta)>0, \lambda_{2}=\lambda_{2}(k, \beta)<0$ and 2 purely complex eigenvalues $\lambda_{3}=i \omega, \lambda_{4}=-i \omega, \omega=\omega(k, \beta)$.

\section{Acknowledgement.}

This research was supported by Horizon2020-2017-RISE-777911 project.

\section{References}

[1] C. J. Amick and J. F. Toland, Homoclinic orbits in the dynamic phase space analogy of an elastic strut, European J. Appl. Math. 3, 97-114 (1992).

[2] M. S. Berger, Nonlinearity and Functional Analysis. Lectures on Nonlinear Problems in Mathematical Analysis (Academic Press, 1977) 417 pp.

[3] H. Brezis and L. Nirenberg, Remarks on finding critical points, Comm. Pure Appl. Math. 44, 939-963 (1991).

[4] B. Buffoni, A. R. Champneys and J. F. Toland, Bifurcation and coalescence of multimodal homoclinic orbits for a Hamiltonian system, J. Dynam. Differential Equations 8 , 221-281 (1996).

[5] S. N. Chow and D. R. Dunninger, A maximum principle for n-metaharmonic functions, Proc. Amer. Math. Soc. 43, 79-83 (1974).

[6] Y. Chen and P. J. McKenna, Traveling waves in a nonlinearly suspended beam: theoretical results and numerical observations, J. Differential Equations 136, 325-355 (1997).

[7] C. - P. Danet, Existence and uniqueness of weak and classical solutions for a fourthorder semilinear boundary value problem, ANZIAM J. 61, 305-319 (2019).

[8] C. - P. Danet, Uniqueness in some higher order elliptic boundary value problems in $n$ dimensional domains, Electronic J. Qualitative Theory of Dif. Eq. 54, 1-12 (2011).

[9] C. - P. Danet and A. Mareno, Maximum principles for a class of linear elliptic equations of even order, Math. Inequal and Appl. 16, 809-822 (2013).

[10] C. - P. Danet, Existence and uniqueness tesults for a class of higher order equations, submitted.

[11] I. Ekeland, Convexity Methods in Hamiltonian Systems (Springer Verlag, 1990) 257 pp.

[12] I. M. Gelfand, Some problems in the theory of quasilinear equations, Amer. Math. Soc. Transl. 29, 295-381 (1963).

[13] D. Gilbarg and N. S. Trudinger, Elliptic Partial Differential Equations of Second Order (Springer Verlag, 2001) 531 pp.

[14] V. Komkov, Certain estimates for solutions of nonlinear elliptic differential equations applicable to the theory of thin plates, SIAM J. Appl. Math. 28, 24-34 (1975).

[15] Y. A. Kuznetsov, Elements of Applied Bifurcation Theory (Springer Verlag, 1995) 533 pp.

[16] B. Kawohl and G. Sweers, On the differential equation $u_{x x x x}+u_{y y y y}=f$ for an anisotropic stiff material, SIAM J. Math. Anal. 37, 1828-1853 (2006).

[17] O. A. Ladyzhenskaya, The Mathematical Theory of Viscous Incompressible Flow (Gordon and Breach Science Publishers, 1969) 224 pp. 
[18] A. C. Lazer and P. J. McKenna, Large-amplitude oscillations in suspension bridges: some new connections with nonlinear analysis, Siam Rev. 32, 537- 78 (1990).

[19] M. A. Peletier, W. C. Troy and Vand der Vorst, Stationary solutions of a fourth-order nonlinear diffusion equation, Differential equations 31, 301-314 (1995).

[20] M. A. Peletier, Sequential buckling: a variational analysis, SIAM J. Math. Anal. 32, 1142-1168 (2001).

[21] M. H. Protter and H. F. Weinberger, Maximum Principles in Differential Equations (Prentice - Hall, Inc., Englewood Cliffs, N. J., 1967) 271 pp.

[22] S. Tersian and J. Chaparova, Periodic and homoclinic solutions of extended FisherKolmogorov equations, J. Math. Anal. Appl. 260, 490-506 (2001).

[23] S. Tersian and J. Chaparova, Periodic and homoclinic solutions of some semilinear sixth - order diferential equations, J. Math. Anal. Appl. 272, 223-239 (2002). 